\title{
Pathological findings coronavirus disease-19
}

\author{
Marco A. Duran-Padilla* \\ Service of Pathology, Hospital General de México "Dr. Eduardo Liceaga”, Mexico City, Mexico
}

In December 2019, patients with pneumonia of unknown origin appeared in Wuhan, China, whose clinical features indicated an acute respiratory syndrome derived from a viral infection. The first three cases were reported as a new virus, the seventh type of the Beta-coronavirus family ${ }^{1}$ that appeared in patients over the age of 60 mainly, who presented with comorbidities such as high blood pressure, obesity, and diabetes, among other diseases.

The World Health Organization (WHO) considers coronavirus disease (COVID-19) as a disease that is clinically asymptomatic or with mild and moderate symptoms. It is a highly contagious airborne disease that can also be transmitted by fomites. At present, the polymerase chain reaction test is the elected means of diagnosis, where samples are collected from the mucosae of the upper respiratory tract, such as the nasopharynx and/or bronchoalveolar lavage, or by serologic tests for the identification of antibodies (Immunoglobulin $M[\lg M]$ and $\lg G)$.

The severe acute respiratory syndrome coronavirus (SARS CoV)-2 virus belongs to the Coronavirus family with an RNA-containing nucleus and a crown-like halo of glycoproteins responsible for binding to receptors of the angiotensin-converting enzyme 2, which has a high expression in endothelial and smooth muscle cells and can spread to virtually all organs causing systemic disease.

On March 11, 2020, the WHO declared it a pandemic ${ }^{2}$. By July 5, 2020, approximately 11.5 million people were infected and 500,000 deaths were reported. By July 22,
617,000 deaths had been reported in 215 countries and 15 million people had already been infected ${ }^{3}$. In November, 57,245,965 patients were diagnosed with COVID-19 with 1,361,419 reported deaths. In Mexico, as of November 20, 2020, the following numbers have been disclosed: 1,019,543 patients have been infected with SARS CoV-2 virus, with 766,361 patients having recovered whereas 100,104 patients have died ${ }^{3}$.

\section{Autopsy and COVID-19}

According to the recommendations of the United States federal agency, Center for Disease Control and Prevention, a complete autopsy in patients who died from COVID-19 should be limited to special cases, since adequate biosecurity measures type III or IV are required with infrastructure that maintains conditions such as negative pressure ventilation, restricted access of personnel, and adequate personal protective. The risk of transmission of the virus from the corpse to medical and paramedical personnel is very high due to aerosol dispersion, fluid spills, contamination of facilities, and work equipment.

Unfortunately, the quality standards required for biosecurity measures in pathology laboratories are not available in many countries. In forensic medical terms, their implementation must be assessed, as regulations generally require the performance of an autopsy.

Trucut biopsies have been performed as a measure to collect tissue under safe conditions. In some institutions in Mexico, multidisciplinary protocols are being
Correspondence:

*Marco A. Duran-Padilla

E-mail: patologiaduran@ hotmail.com

DOI: 10.24875/HGMX.M21000039 NC-ND license (http://creativecommons.org/licenses/by-nc-nd/4.0/) de Mexico. Published by Permanyer. er. This is an open access article under the CC BY-

Available online: 22-01-2021 Rev Med Hosp Gen Mex. 2021;84(1):1-3 www.hospitalgeneral.mx
article under the CC BY- 
carried out to take samples by means of ultrasound-guided needle biopsy from different organs. Some countries have already reported morphological results, although the number of patients has been limited. Likewise, in cytopathology, cellular studies and fine-needle aspiration biopsies have been performed in patients with COVID-19. One of the largest studies ever conducted in the literature, concerning postmortem studies, reported 68 autopsied patients from Italy and New York ${ }^{4}$, where a wide spectrum of histological lesions in the lung was reported, affecting both the epithelium and vessels of other organs.

\section{Macroscopic pulmonary findings}

No macroscopic patterns characteristic of COVID-19 have been described. However, changes may be similar to those observed in patients with diseases produced by infectious processes, terminal illnesses associated with diffuse alveolar damage (DAD) or obstructive shock. Alterations are mainly associated with edema and weight gain (over $1300 \mathrm{~g}$ ), with diffuse and patchy consolidation in the lung parenchyma, mucosal ulceration of the upper airways, even in cases where patients have not been intubated, necrotizing bronchiolitis, hemorrhage, embolism as well as acute purulent and chronic pleurisy that may be secondary to collective infection by bacteria, fungi, and viruses.

\section{Microscopic pulmonary findings}

Histopathological changes in COVID-19 are the most prominent, although not specific compared to other organs. The most significant pulmonary data as in other viral infections are DAD.

In most cases studied, lesions are epithelial, such as hyperplasia of type II pneumocytes and desquamative interstitial pneumonia. Occasionally, basophilic intracytoplasmic inclusions are observed in the hyperplastic cells that may correspond to viral inclusion bodies. These are not commonly found by optical microscopy and are demonstrated by electron microscopy. There is presence of epidermoid metaplasia of the epithelium when the evolution of the condition is chronic. Vascular damage is produced by microthrombi, fibrinoid necrosis, and deep vein thrombosis. There are variable degrees of fibroblast proliferation and collagen deposition with dense and myxoid fibrotic reaction patterns ${ }^{4}$. Three stages have been described according to the evolution of the disease:

\section{Exudative stage}

This consists of the formation of hyaline membranes, protein exudate, cell desquamation, alveolar bleeding, and fibrinoid necrosis of small-caliber vessels.

\section{Organizational stage}

There is fibrin deposition, cell proliferation and hyperplasia mainly of pneumocytes and fibroblasts, and chronic infiltration of lymphocytes.

\section{Fibrotic stage}

There is deposition of dense collagen fibers in the interstitium with some mononuclear cell infiltrates.

In any of the stage, there can be additional etiological agents, such as herpes virus, cytomegalovirus with intranuclear inclusions and/or bacterial or mycotic diseases.

These pathological changes are very similar to those reported in the epidemiological emergency of the virus. In November 2009, 15 autopsies were performed in a postmortem study conducted at the Pathology Service of the General Hospital of Mexico Dr. Eduardo Liceaga, during the $\mathrm{H} 1 \mathrm{~N} 1{ }^{5}$ epidemic. These autopsies revealed that histopathological changes due to DAD were described as representative of viral infection, but not pathognomonic. This is similar to what has been described in COVID-19 infection with hyaline membrane formation, hyperplasia of pneumocytes, fibrin thrombi occluding the lumen, bronchial wall necrosis, edema, hemorrhage, and other non-specific changes, representing a morphological spectrum similar to this family of Coronaviruses.

\section{Extrapulmonary pathological findings}

Other microscopic findings in different organs taken from punch biopsies and autopsy studies have been described 6 :

\section{Cardiovascular system}

Myocardial edema, endotheliitis, lymphocytic infiltration, serosanguinous pericardial effusion, and microthrombosis.

\section{Liver}

Steatosis, spotty necrosis, Kupffer cell hyperplasia, and lymphocytic infiltrate distributed in the portal spaces and some sinusoids and microthrombi. 


\section{Kidney}

Acute tubular necrosis of varying degrees of the proximal tubules with vacuolation and hemosiderin pigment, interstitial lymphocytic infiltrate, and microangiopathy with thrombosis. Changes in the glomeruli are mesangial expansion and arteriolar hyalinosis. Rare cases of collapsing glomerulopathy have also been reported.

\section{Gastrointestinal tract}

Epithelial necrosis, endotheliitis, and ischemic colitis.

\section{Bone marrow and lymph nodes}

Nodular histiocytic hyperplasia with hemophagocytosis.

\section{Nervous system}

Ischemic necrosis, hemorrhagic infarct, edema, microthrombosis, and demyelination.

\section{Skin}

Histological changes correspond to lesions such as hives, blistering lesions, and rashes that are not characteristic of COVID-19 infection.

Regarding ultrastructural changes, viral particles have been identified with transmission electron microscopy, measuring between 60 and $140 \mathrm{~nm}$ and spikes of 9 and $12 \mathrm{~nm}$. These extracellular viral particles and inclusion bodies remain in the epithelial cell membrane vesicles of the respiratory system.
Pathological studies such as biopsy, autopsy, and cytology with their respective immunohistochemical markers, ultrastructure, and in situ hybridization are very valuable tools for understanding the natural history of the disease in correlation with the clinical manifestations revealed by imaging and genetic studies.

Today, the mortality and morbidity of this virus have shifted expectations in terms of global healthcare and economic conditions of society. The world community, medicine, and science are working toward delivering a specific treatment against the SARS CoV-2 virus, with great efficacy and safety, as well as a cost-effective vaccine, which will counteract the spread and new gene mutations of the virus regarding this pandemic. Working with institutional authorities is necessary, to develop specific protocols that allow for autopsies, biopsies, and cytological studies to be carried out in proper conditions to promote and generate greater knowledge about this new disease.

\section{References}

1. Zhu N, Zhang D, Wang W, Li X, Yang B, Song J, et al. A novel coronavirus from patients with pneumonia in China, 2019. $\mathrm{N}$ Engl J Med. 2020;382:727-33.

2. World Health Organization. 2020. Available from: https://www.who.int/ news-room/detail/30-01-2020-statement-on-the-second-meeting-ofthe-internationqal-health-regulations-(2005)-emergency-committee-regarding-the-outbreak-of-novel-coronavirus-(2019-ncov).

3. COVID-19 Dashboard by the Center for Systems Science and Engineering (CSSE) at Johns Hopkins University (JHU); 2020. Available from: https://www.arcgis.com/apps/opsdashboard/index.html\#/bda7594740fd40299423467b48e9ecf6. [Last accessed on 2020 Nov 20].

4. Borczuk A, Salvatore S, Seshan S, Patel S, Bussel JB, Mostyka M, et al. COVID-19 pulmonary pathology: a multi-institutional autopsy cohort from Italy and New York city. Mod Pathol. 2020;33:2156-68.

5. Soto-Abraham MV, Soriano-Rosas J, Diaz-Quilonez A, Silva-Pereyra J, Vazquez-Hernandez $\mathrm{P}$, Torres-Lopez $\mathrm{O}$, et al. Pathological changes associated with the $2009 \mathrm{H} 1 \mathrm{N1}$ virus. N Engl J Med. 2009;361:2001-3.

6. Vazquez-Bonilla WO, Orozco R, Argueta V, Sierra M, Zambrano LI, Muñoz-Lara $F$, et al. A review of the main histopathological findings in coronavirus disease 2019. Hum Pathol. 2020;105:74-83. 